\title{
MATRICARIETUM DISCOIDEO-RECUTITAE JAROLÍMEK ET AL. 1997 AND MATRICARIO-ANTHEMIDETUM COTULAE DIHORU 1975 EX MUCINA 1987 - TWO POORLY KNOWN ASSOCIATIONS FROM THE MALVION NEGLECTAE (GUTTE 1966) HEJNÝ 1978 ALLIANCE IN RURAL AREAS OF NORTH-WEST POLAND
}

\author{
Andrzej Brzeg, Katarzyna Szrama, Maria Wojterska
}

\begin{abstract}
A. Brzeg, K. Szrama, M. Wojterska, Department of Plant Ecology and Environmental Protection, Institute of Environmental Biology, Faculty of Biology, Adam Mickiewicz University, Umultowska 89, 61-614 Poznań, Poland, e-mail: mwzerios@amu.edu.pl
\end{abstract}

(Received: December 10, 2013. Accepted: January 27, 2014)

\begin{abstract}
Studies were conducted in 50 villages of mediaeval origin in NW Poland. In eight of them were found patches of two Malvion neglectae associations: Matricarietum discoideo-recutitae and Matricario-Anthemidetum cotulae. The documentation comprising 19 phytosociological relevés was made according to the Braun-Blanquet approach in the years 2011-2013. Only one relevé dates from 2006 and was made in N Wielkopolska. Two new subassociations: Matricarietum discoideo-recutitae medicaginetosum lupulinae and M. d.-r. typicum were described. As a result of decline of traditional forms of management in villages both associations, and especially Matricario-Anthemidetum cotulae, are contemporarily strongly endangered.
\end{abstract}

KEY WORDS: ruderal vegetation, Malvion neglectae, rural areas, Pomerania, Lubuskie Lakeland, North-West Poland

\section{INTRODUCTION}

Data concerning the differentiation of vegetation of settlements in the rural landscapes of NW Poland were collected in the course of studies on their plant cover, conducted within the grant of NSC NN 305 062440. Out of numerous interesting ruderal communities worth special attention are two associations from the Malvion neglectae alliance, either not yet documented in Poland, or very poorly known. The aim of this paper is to give the first phytosociological documentation for these syntaxa from NW Poland.

\section{MATERIAL AND METHODS}

The documentation comprises 19 phytosociological relevés, made according to the BRAUN-BLANQUET (1964) approach in the years 2011-2013. Studied patches were found in eight out of 50 villages of mediaeval origin (WojtersKA et al. 2007) in NW Poland
(Fig. 1). Only one relevé dates from 2006 and was made in N Wielkopolska. All relevés were compiled in an analytical table. The distribution of villages, in which studied communities were documented, is shown in Figure 1, on the background of ATPOL (ZAJĄC 1978) grid.

The nomenclature of vascular plants follows Mirek et al. (2001), and only some taxa, mainly of lower rank, are named after RUTKOwski (2004). Names of mosses follow Ochyra et al. (2003). Diagnostic role of species and syntaxonomical appurtenance of examined communities was accepted after JAROLÍMEK et al. (1997) and RATYŃSKA et al. (2010).

In order to describe the major directions of variation of studied communities, the relevé data underwent principal component analysis (PCA). Mean Ellenberg indicator values (EIV, ELLENBERG et al. 1992) were included in the analysis as passive variables. The calculation and graphic presentation of results was made with help of Canoco 4.5 SOFTWARE (ter BRAAK \& Šmilauer 2002). 


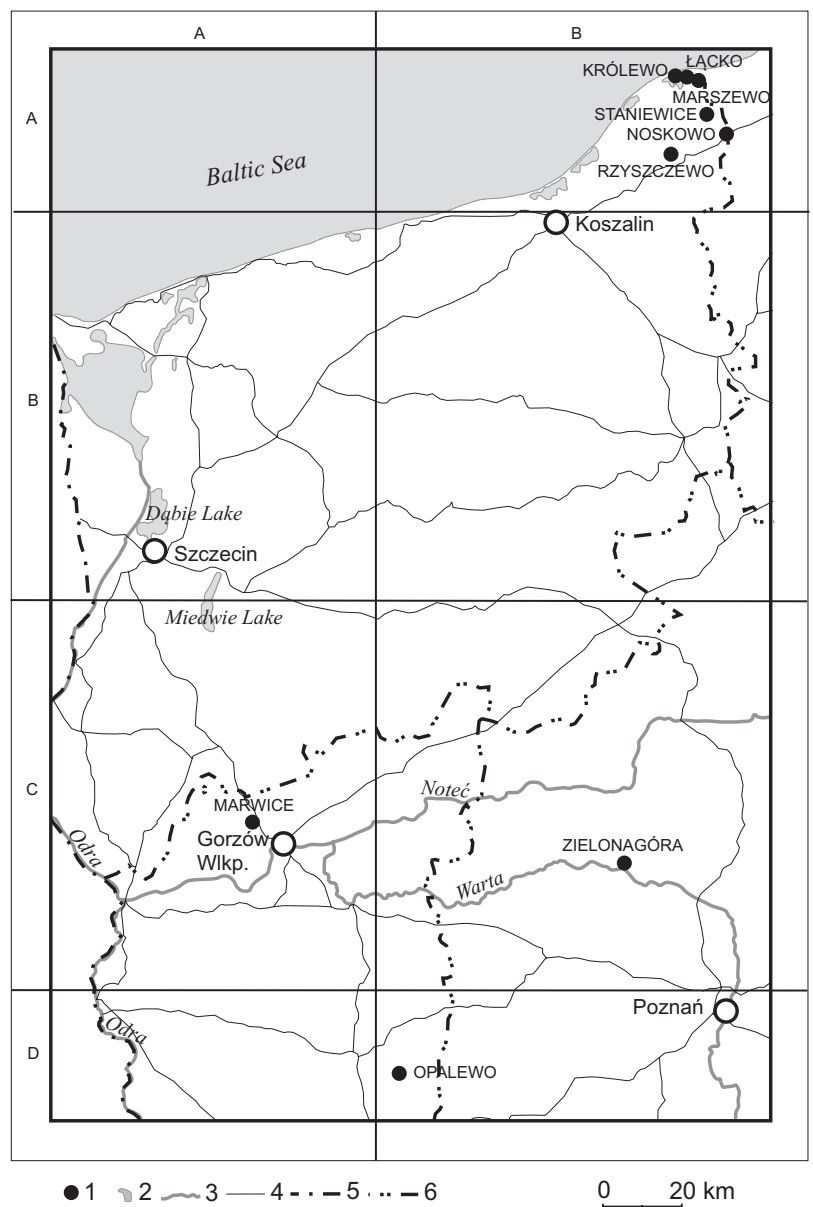

Fig. 1. Distribution of villages, in which the studied communities were documented, on the background of ATPOL grid: 1 - villages, 2 - water bodies, 3 - rivers, 4 - main roads, 5 - state boundary, 6 - boundaries of voivodeships

\section{RESULTS}

\section{Syntaxonomy}

Cl.: Stellarietea mediae R. Tx. et al. in R. Tx. 1950

O.: Sisymbrietalia J. Tx. in Lohmeyer et al. 1962

All.: Malvion neglectae (Gutte 1966) Hejný 1978

Ass.: Matricarietum discoideo-recutitae

Jarolímek et al. 1997

Matricario-Anthemidetum cotulae Dihoru

1975 ex Mucina 1987

\section{Characteristics of syntaxa}

Matricarietum discoideo-recutitae (Table 1, rel. 1-12) is a ruderal community connected with settlements and their direct surroundings. Its patches occurred on road margins in the villages and among adjacent fields, as well as on recently disturbed areas, like evened ruins of farm buildings and construction places. Some patches developed in abandoned vegetable gardens. The phytocoenoses are floristically differentiated counting from 26 up to 77 taxa (medium 46). To the group of diagnostic species belong, except for Chamomilla recutita, characteristic and dominating in the majority of patches, transgressive taxa from segetal communities. The most numerous are taxa characteristic for the order Sisymbrietalia and for the class Stellarietea mediae. Plants connected with treading, from both Polygono-Poetea annuae and Molinio-Arrhenatheretea classes, constitute constant element of the floristic structure of this unit. Perennial ruderal species of the Artemisietea vulgaris class are quite numerous, but with very low abundance and vitality. Physiognomy of phytocoenoses is mainly determined by two species of mayweeds (Figs. 2 and 3).

The authors of the first description of association (JAROLÍMEK et al. 1997) have not divided it into any units of lower rank. Our Table 1 shows yet distinct differentiation of Matricarietum discoideo-recutitae in two units, which we propose to rank as subassociations.

The first of them (rel. 1-4, Fig. 2) stands out with very high floristic richness (medium 71 taxa). All of these plots occurred in abandoned state farms, or in granges, on newly disturbed substrate, containing

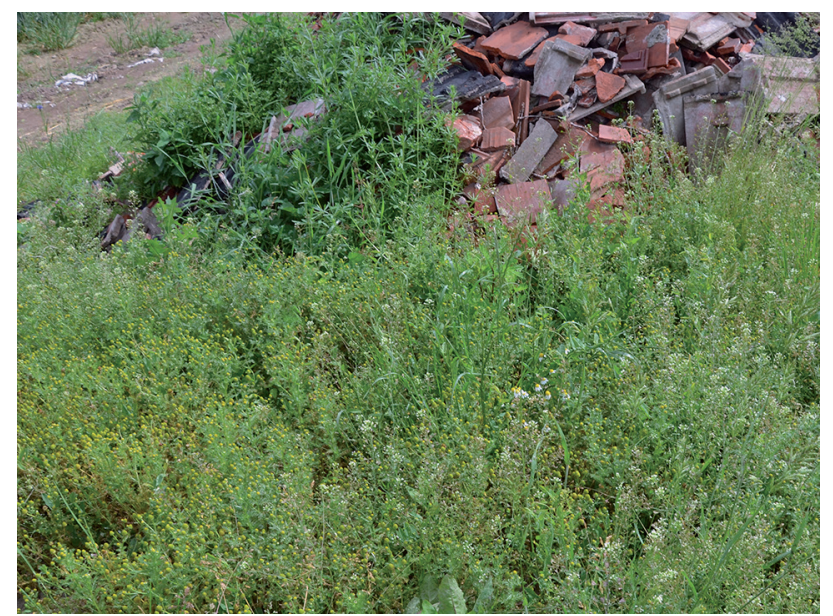

Fig. 2. Fragment of the patch of Matricarietum discoideo-recutitae medicaginetosum lupulinae in Rzyszczewo (phot. M. Wojterska)

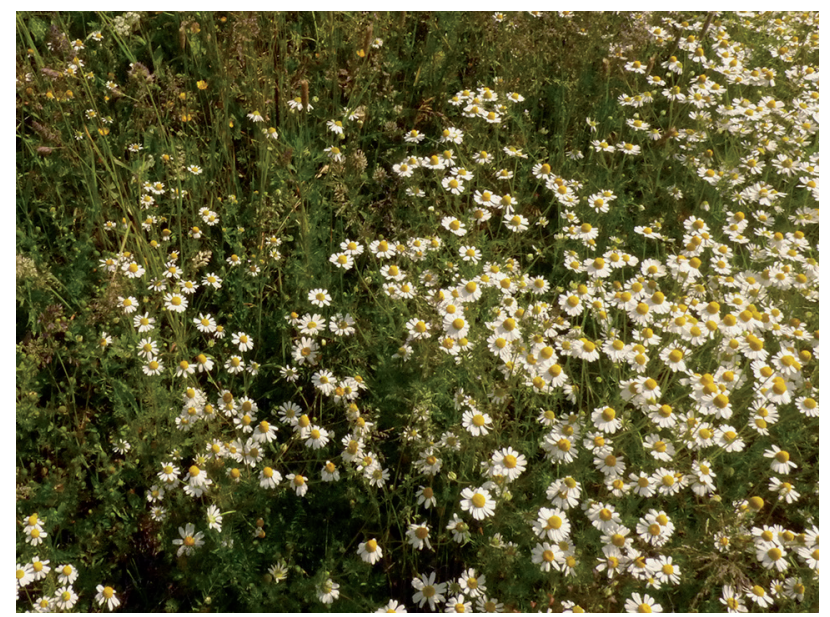

Fig. 3. Fragment of the patch of Matricarietum discoideo-recutitae typicum in Opalewo (phot. M. Wojterska) 
rubble and mortar. They were found on big surfaces (reaching more than $100 \mathrm{~m}^{2}$ ) in two villages: Królewo and Rzyszczewo.

\section{Syntaxonomical description}

Matricarietum discoideo-recutitae medicaginetosum lupulinae subass. nova hoc loco

Nomenclatural type: Table 1, rel. 3 (oryg.) holotypus hoc loco

Differential taxa: Barbarea vulgaris, Bryum argenteum, Cerastium holosteoides, Cirsium vulgare, Medicago lupulina, Plantago lanceolata, Poa pratensis s.s., Ranunculus repens, Rumex crispus, Vicia angustifolia, Vicia tetrasperma.

The second, more widespread, typical subassociation (rel. 5-12, Fig. 3) is less rich in species (medium 34 taxa). The examined plots occurred on road margins in the village and among neighbouring fields and covered smaller areas. They were documented from six villages: Łącko, Marszewo, Noskowo, Opalewo, Staniewice and Zielonagóra, as well as noted in several other localities. As regionally differential species can be mentioned: Centaurea cyanus, Erysimum cheiranthoides, Galinsoga parviflora and Polygonum persicaria.

\section{Syntaxonomical description}

Matricarietum discoideo-recutitae typicum subass. nova hoc loco

Nomenclatural type: Table 11, rel. 14 (JAROLímeK et al. 1997: 164-165) holotypus hoc loco, typus subass. et ass.

The association has been stated so far only in Slovakia (JAROLÍMEK et al. 1997), NE Slovenia (ČARNI 2005), and Hungary (BorHIDI 2007). From Poland it has been mentioned in the list of plant associations of Wielkopolska (Brzeg \& WojtersKa 2001) on the basis of unpublished data.

Matricario-Anthemidetum cotulae (Table 1, rel. 13-19) is a rare ruderal community (RATYŃSKA et al. 2010). In the study area its patches were found with-

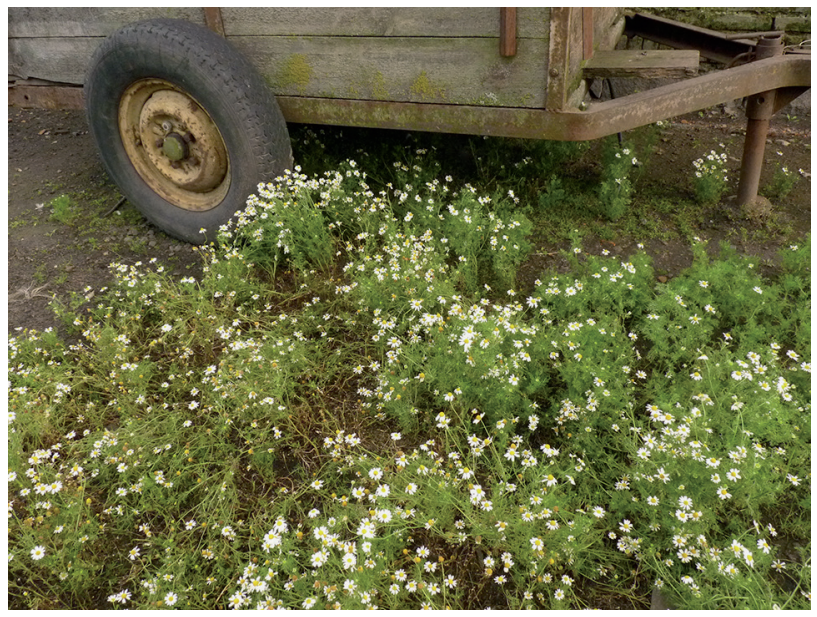

Fig. 4. The patch of Matricario-Anthemidetum cotulae in the poultry yard in Marwice (phot. M. Wojterska) in villages on extensively trodden poultry-yards. The floristic composition is stable (25-31 taxa, medium 28 per relevé). The diagnostic species are: Anthemis cotula (dominating characteristic species, Fig. 4 and 5) and Potentilla anserina (differential towards Matricarietum discoideo-recutitae). The association does not show distinct variability. Besides typical form (rel. 13-17), two patches are differentiated by hygrophilous plants: Juncus bufonius, Polygonum hydropiper and other Bidentetea tripartitae species (rel. 18-19). This partly confirms the variability within association, suggested by BRZEG \& RosADZIŃsKi (2006).

The syntaxon has been not validly described from Rumania (DiHoru 1975) and validated by Mucina (1987). Further documentation origins from Slovakia (Mucina 1987, JarolímeK et al. 1997), Germany (Passarge 1996, Schubert et al. 2001) and Poland (PAWlak 1992, Brzeg \& Rosadziński 2006). Sowa, already in 1971 (p. 161), noticed in central Poland dense aggregations of Anthemis cotula, and indicated the necessity of their closer examination. The association is in NW Poland very rare, similarly to its characteristic species, only sporadically noted there in the contemporary floristic studies (e.g. TRUCHAN \& SoBIsz 2007).

Principal Component Analysis (PCA, Fig. 6) shows both distinct differences between associations Matricario-Anthemidetum cotulae and Matricarietum discoideo-recutitae and subassociations within the second unit.

Passive projection of mean EIV onto the PCA ordination diagram (Fig. 7) shows that relevés distributed along first ordination axis differ in soil productivity and reaction requirements of plants constituting their floristic combinations. The species growing in the Matricarietum discoideo-recutitae medicaginetosum show lower requirements to soil fertility than those growing in Matricarietum discoideo-recutitae typicum and Matricario-Anthemidetum cotulae.

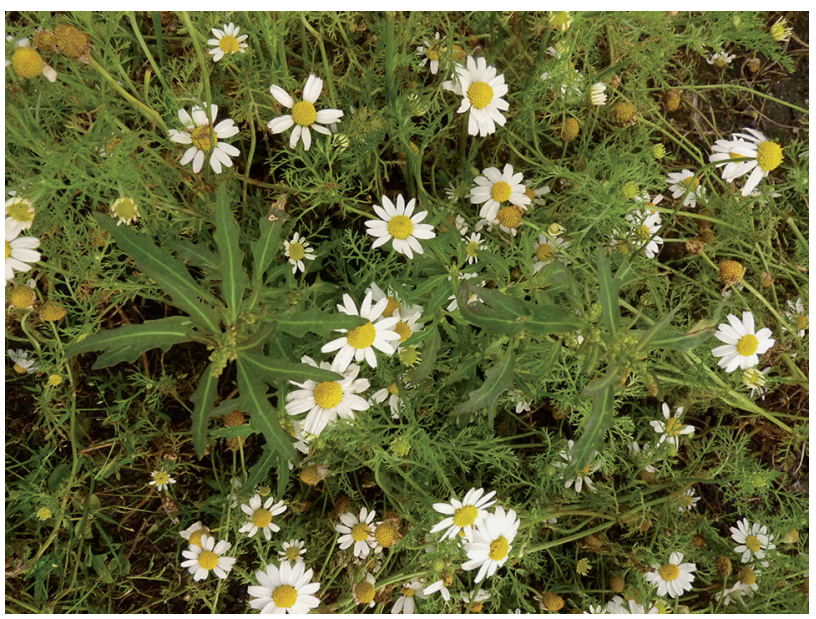

Fig. 5. Close-up of Matricario-Anthemidetum cotulae with participation of Chenopodium glaucum in Marwice (phot. M. Wojterska) 
Table 1. Floristic composition and differentiation of Matricarietum discoideo-recutitae (rel. 1-12) and Matricario-Anthemidetum cotulae (rel. 13-19)

\begin{tabular}{|c|c|c|c|c|c|c|c|c|c|c|c|c|c|c|c|c|c|c|c|c|c|}
\hline No of relevé & 1 & 2 & 3 & 4 & 5 & 6 & 7 & 8 & 9 & 10 & 11 & 12 & & 13 & 14 & 15 & 16 & 17 & 18 & 19 & \\
\hline Locality & $\mathrm{K}$ & K & $\mathrm{K}$ & $\mathrm{Rz}$ & $\mathrm{N}$ & Op & $\mathrm{E}$ & Ms & Ms & $\mathrm{St}$ & $\mathrm{Zg}$ & Op & \multirow{10}{*}{ 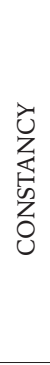 } & Ms & Ms & E & $\mathrm{Mw}$ & $€$ & 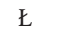 & $\mathrm{K}$ & \multirow{10}{*}{ 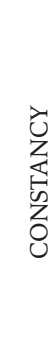 } \\
\hline (day) & 11 & 11 & 11 & 09 & 07 & 14 & 12 & 12 & 12 & 27 & 09 & 14 & & 12 & 12 & 12 & 10 & 12 & 12 & 11 & \\
\hline Date (month) & 07 & 07 & 07 & 06 & 06 & 06 & 07 & 07 & 07 & 05 & 06 & 06 & & 07 & 07 & 07 & 08 & 07 & 07 & 07 & \\
\hline (year) & 11 & 11 & 11 & 12 & 12 & 13 & 11 & 11 & 11 & 12 & 04 & 13 & & 11 & 11 & 11 & 11 & 11 & 11 & 11 & \\
\hline Aspect & & & & & & & & & SW & & & & & & & & & & & & \\
\hline Inclination $\left[^{\circ}\right]$ & & & & & & & & & 3 & & & & & & & & & & & & \\
\hline Cover of herb layer c [\%] & 90 & 80 & 70 & 70 & 60 & 80 & 75 & 75 & 80 & 75 & 85 & 85 & & 90 & 90 & 80 & 70 & 70 & 75 & 90 & \\
\hline Cover of moss layer d [\%] & 10 & 10 & + & 20 & - & + & - & - & - & - & - & - & & - & - & + & + & + & 5 & 10 & \\
\hline Area of relevé $\left[\mathrm{m}^{2}\right]$ & 25 & 25 & 20 & 15 & 50 & 6 & 12 & 10 & 14 & 10 & 6 & 6 & & 15 & 8 & 25 & 12 & 10 & 20 & 9 & \\
\hline Number of taxa & 77 & 71 & 72 & 64 & 40 & 33 & 41 & 32 & 39 & 26 & 32 & 30 & & 31 & 25 & 25 & 29 & 26 & 27 & 30 & \\
\hline \multicolumn{22}{|l|}{ I. Ch., ${ }^{*}$ D. Ass. } \\
\hline Chamomilla recutita & 4.4 & 4.4 & 3.4 & 4.4 & 2.3 & 3.4 & 4.4 & 3.4 & 3.4 & 3.1 & 4.4 & 1.2 & $\mathrm{~V}$ & 1.1 & + & + & . & + & 2.2 & . & IV \\
\hline *Veronica persica & + & + & + & . & + & 1.2 & + & + & 1.1 & + & +.2 & . & $\mathrm{V}$ & . & . & . & . & . & . & . & - \\
\hline *Vicia hirsuta & 1.1 & + & 1.1 & + & $\mathrm{r}$ & $+^{\circ}$ & +.2 & . & . & . & + & . & IV & . & . & . & . & . & . & . & - \\
\hline *Veronica arvensis & + & . & . & . & + & +.2 & . & 1.1 & + & . & + & . & III & . & . & . & . & . & . & . & - \\
\hline Anthemis cotula & . & . & . & . & . & . & . & . & . & . & . & . & & 4.4 & 3.2 & 4.4 & 3.4 & 2.2 & 4.4 & 3.4 & $\mathrm{~V}$ \\
\hline *Potentilla anserina & . & . & . & . & . & . & +.2 & . & . & . & . & . & I & & +.2 & 1.2 & + & +.2 & + & 2.3 & $\mathrm{~V}$ \\
\hline \multicolumn{22}{|l|}{ II. Ch., *D. Malvion neglectae } \\
\hline *Galinsoga ciliata & . & . & . & . & . & . & . & + & + & . & . & + & II & 1.1 & $\mathrm{r}$ & . & . & $\mathrm{r}$ & . & . & III \\
\hline Urtica urens & . & . & . & . & . & . & . & . & . & . & 2.1 & . & I & . & + & $\mathrm{r}$ & . & . & +.2 & . & III \\
\hline Aethusa cynapium s.s. & + & + & $+^{\circ}$ & . & . & . & . & . & . & . & . & . & II & . & . & $\mathrm{r}^{\circ}$ & . & . & . & . & I \\
\hline${ }^{*}$ Chenopodium polyspermum & + & + & + & . & . & . & . & . & + & . & . & . & II & . & . & . & . & . & . & . & - \\
\hline Malva neglecta & . & . & . & . & . & +.2 & . & . & . & . & +.2 & . & $\mathrm{I}$ & . & . & . & . & . & . & . & - \\
\hline \multicolumn{22}{|l|}{ III. Ch. Sisymbrietalia } \\
\hline Sisymbrium officinale & . & + & $\mathrm{r}$ & $\mathrm{r}$ & . & 1.3 & + & 1.1 & + & . & 1.1 & . & IV & + & + & 1.1 & . & 1.1 & . & . & III \\
\hline Atriplex patula & + & . & + & . & . & + & . & + & + & . & + & + & III & + & 1.1 & . & . & + & . & . & III \\
\hline Chenopodium album var. lanceolatiforme & 2.1 & + & 1.1 & . & . & . & + & + & + & + & . & . & III & . & + & . & . & + & . & + & III \\
\hline Conyza canadensis & 1.1 & . & + & + & + & + & $\mathrm{r}$ & . & . & . & + & + & IV & . & . & . & + & . & . & . & I \\
\hline Lactuca serriola & . & + & $\mathrm{r}$ & + & . & . & . & . & $\mathrm{r}$ & . & . & + & III & . & . & . & . & . & . & . & - \\
\hline Descurainia sophia & . & . & . & . & $\mathrm{r}$ & $\mathrm{r}^{\circ}$ & . & . & . & . & . & $\mathrm{r}$ & II & . & . & . & . & . & . & . & - \\
\hline \multicolumn{22}{|l|}{ IV. Ch. Stellarietea mediae } \\
\hline Capsella bursa-pastoris var. integrifolia & 2.1 & 2.1 & 2.1 & 1.1 & 2.1 & 2.1 & + & 1.1 & 1.1 & 1.1 & 1.1 & 2.3 & $\mathrm{~V}$ & + & 1.1 & 1.1 & . & + & + & + & $\mathrm{V}$ \\
\hline Chenopodium album s.s. & 2.1 & 1.1 & 1.1 & . & 2.1 & 1.1 & 1.1 & 1.1 & 1.1 & 2.1 & 1.1 & + & $\mathrm{V}$ & 2.2 & 2.1 & . & + & + & $\mathrm{r}^{\circ}$ & + & $\mathrm{V}$ \\
\hline Matricaria perforata & 2.2 & 2.2 & 2.1 & + & 2.2 & 1.1 & 2.2 & 2.1 & 2.1 & . & . & . & IV & + & +.2 & + & 2.1 & . & 1.2 & . & IV \\
\hline Stellaria media & + & + & + & + & $\mathrm{r}^{\circ}$ & 1.2 & . & . & + & +.2 & . & +.2 & IV & + & . & $+^{\circ}$ & $\mathrm{r}$ & . & + & 1.1 & IV \\
\hline Polygonum aviculare s.s. & 1.1 & 1.1 & 1.1 & . & + & . & 1.1 & 2.1 & 2.1 & . & . & + & IV & + & 1.2 & . & . & . & . & . & II \\
\hline Apera spica-venti & 1.1 & 1.2 & + & . & . & 1.1 & + & + & $\mathrm{r}$ & . & . & . & III & +.2 & + & . & . & . & . & + & III \\
\hline Geranium pusillum & + & + & + & + & + & 2.2 & . & . & . & . & +.2 & + & IV & . & . & . & . & . & + & . & I \\
\hline Fallopia convolvulus & + & $\mathrm{r}$ & + & . & $\mathrm{r}$ & . & . & . & $\mathrm{r}$ & 1.2 & . & . & III & $\mathrm{r}$ & . & . & . & . & . & + & II \\
\hline Centaurea cyanus & + & . & . & . & . & + & + & + & . & $(+)$ & . & . & III & + & . & . & . & . & . & . & I \\
\hline Polygonum persicaria & $\mathrm{r}$ & . & . & . & . & $+^{\circ}$ & + & . & . & + & . & . & II & + & . & . & + & . & . & . & II \\
\hline Galinsoga parviflora & . & . & . & . & . & . & . & $\mathrm{r}$ & $\mathrm{r}$ & . & 1.2 & + & II & . & . & . & + & . & . & . & I \\
\hline Sonchus asper & . & . & . & . & . & . & . & + & + & . & . & . & I & $\mathrm{r}$ & . & $\mathrm{r}$ & . & . & + & . & III \\
\hline Echinochloa crus-galli & . & . & . & . & . & $\mathrm{r}$ & . & . & . & . & + & . & I & 1.1 & + & . & . & . & . & . & II \\
\hline Erysimum cheiranthoides & . & . & . & . & $\mathrm{r}$ & . & . & . & . & $(+)$ & $\mathrm{r}$ & . & II & . & . & . & . & . & . & . & - \\
\hline Lamium purpureum & + & . & $\mathrm{r}$ & . & $\mathrm{r}$ & 1.2 & . & . & . & . & + & . & III & . & . & . & . & . & . & . & - \\
\hline Myosotis arvensis & + & + & + & $\mathrm{r}$ & . & + & . & . & . & . & . & . & III & . & . & + & . & . & . & . & I \\
\hline Senecio vulgaris & . & + & $\mathrm{r}$ & r & + & . & . & . & . & . & + & . & III & . & . & . & . & . & + & . & I \\
\hline Thlaspi arvense & . & + & + & $\mathrm{r}$ & + & . & . & . & . & + & . & . & III & . & . & . & . & . & . & . & - \\
\hline Sonchus oleraceus & $\mathrm{r}$ & + & . & . & . & . & + & . & . & . & . & + & II & . & . & . & . & . & . & . & - \\
\hline Viola arvensis & + & + & . & + & $\mathrm{r}$ & 1.2 & . & . & . & . & . & . & III & . & . & . & . & . & . & . & - \\
\hline Polygonum lapathifolium ssp. pallidum & $\mathrm{r}$ & $\mathrm{r}$ & $\mathrm{r}$ & . & . & . & . & . & . & . & . & . & II & + & . & . & . & . & . & . & I \\
\hline Vicia angustifolia & + & + & + & $\mathrm{r}$ & . & . & . & . & . & . & . & . & II & . & . & . & . & . & . & . & - \\
\hline Vicia tetrasperma & + & + & . & +.2 & . & . & . & . & . & . & . & . & II & . & . & . & . & . & . & . & - \\
\hline Arabidopsis thaliana & . & $\mathrm{r}$ & . & $\mathrm{r}$ & + & . & . & . & . & . & . & . & II & . & . & . & . & . & . & . & - \\
\hline Polygonum monspeliense & . & . & . & . & $\mathrm{r}$ & . & . & . & + & + & . & . & II & . & . & . & . & . & . & . & - \\
\hline V. Ch. Polygono-Poetea annuae & & & & & & & & & & & & & & & & & & & & & \\
\hline Chamomilla suaveolens & 2.1 & 2.1 & 2.1 & 2.2 & 3.4 & 3.4 & 1.2 & 2.1 & 3.4 & 2.4 & 2.1 & 5.4 & $\mathrm{~V}$ & 1.1 & 3.4 & 3.1 & + & 3.2 & 2.1 & 2.1 & $\mathrm{~V}$ \\
\hline Poa апnua & + & + & + & +.2 & 2.1 & 1.2 & + & 1.2 & +.2 & 2.4 & 1.1 & +.2 & $\mathrm{~V}$ & 1.1 & 1.1 & 1.2 & 1.2 & . & 1.2 & 2.3 & $\mathrm{~V}$ \\
\hline Polygonum arenastrum & . & + & +.2 & . & . & + & . & 1.2 & 1.2 & +.2 & 1.2 & + & IV & 2.1 & 2.2 & 2.2 & 1.2 & 2.2 & 1.2 & +.2 & $\mathrm{~V}$ \\
\hline Capsella bursa pastoris var. pinnatifida & + & + & + & . & + & . & + & . & + & $\mathrm{r}$ & . & 1.1 & IV & . & . & $+^{\circ}$ & $\mathrm{r}$ & + & + & . & III \\
\hline Bryum argenteum & 1.2 & 1.2 & + & 1.2 & . & . & . & . & . & . & . & . & II & . & . & +.2 & 1.2 & +.2 & 1.2 & 2.3 & IV \\
\hline Sagina procumbens & . & . & . & . & . & . & . & . & . & . & . & . & - & . & . & . & + & . & 1.2 & . & II \\
\hline Lepidium ruderale & . & . & . & . & . & . & . & . & . & . & . & . & - & . & . & . & +.2 & . & . & . & I \\
\hline
\end{tabular}


Table 1. cont.

\begin{tabular}{|c|c|c|c|c|c|c|c|c|c|c|c|c|c|c|c|c|c|c|c|c|c|}
\hline No of relevé & 1 & 2 & 3 & 4 & 5 & 6 & 7 & 8 & 9 & 10 & 11 & 12 & & 13 & 14 & 15 & 16 & 17 & 18 & 19 & \\
\hline \multicolumn{22}{|c|}{ VI. Ch. Molinio-Arrhenatheretea } \\
\hline Plantago major & 1.1 & 1.1 & 1.1 & 1.1 & 1.1 & +.2 & 1.2 & 1.1 & 2.1 & + & $\mathrm{r}$ & +.2 & $\mathrm{~V}$ & 1.2 & 1.2 & $+^{\circ}$ & 1.1 & + & $+^{\circ}$ & 2.1 & $\mathrm{~V}$ \\
\hline Lolium perenne & +.2 & +.2 & +.2 & +.2 & +.2 & . & 1.2 & 2.1 & +.2 & + & . & 1.2 & $\mathrm{~V}$ & +.2 & +.2 & . & . & 1.2 & . & + & III \\
\hline Trifolium repens & + & + & + & 2.2 & + & . & +.2 & 1.2 & 1.2 & . & . & . & IV & $\mathrm{r}$ & . & +.2 & +.2 & +.2 & +.2 & +.2 & $\mathrm{~V}$ \\
\hline Poa trivialis & + & 1.1 & + & 2.1 & +.2 & $+^{\circ}$ & . & + & +.2 & . & . & +.2 & IV & +.2 & +.2 & . & + & . & . & 1.2 & III \\
\hline Taraxacum officinale agg. & . & . & . & + & $\mathrm{r}$ & . & 1.2 & . & . & . & +.2 & . & II & + & . & . & + & 2.2 & . & + & III \\
\hline Bromus hordeaceus & . & +.2 & . & +.2 & . & 1.2 & . & . & . & . & . & + & II & . & . & . & . & . & $+^{\circ}$ & . & I \\
\hline Ranunculus repens & $+^{\circ}$ & $+^{\circ}$ & + & +.2 & . & . & $\mathrm{r}^{\circ}$ & . & . & . & . & . & III & . & + & . & . & . & $\mathrm{r}$ & +.2 & III \\
\hline Plantago lanceolata & $\mathrm{r}$ & + & + & +.2 & . & . & . & . & . & . & . & . & II & . & . & . & $\mathrm{r}$ & 1.2 & . & . & II \\
\hline Poa pratensis & $\mathrm{r}$ & $\mathrm{r}$ & $+^{\circ}$ & 1.1 & . & . & . & . & . & . & . & . & II & . & +.2 & . & . & +.2 & . & . & II \\
\hline Cerastium holosteoides & $\mathrm{r}$ & + & + & + & . & . & . & . & . & . & . & . & II & . & . & . & . & . & $+^{\circ}$ & . & I \\
\hline Trifolium pratense & +.2 & + & + & . & . & . & . & . & . & . & . & . & II & . & . & . & . & + & . & . & I \\
\hline Rumex crispus & $\mathrm{r}^{\circ}$ & $\mathrm{r}^{\circ}$ & $\mathrm{r}^{\circ}$ & + & . & . & . & . & $\mathrm{r}^{\circ}$ & . & . & . & III & . & . & . & . & . & . & . & - \\
\hline Holcus lanatus & $+^{\circ}$ & . & + & + & . & . & $+^{\circ}$ & . & . & . & . & . & II & . & . & . & . & . & . & . & - \\
\hline Achillea millefolium & . & $+^{\circ}$ & + & . & . & . & . & . & . & . & $\mathrm{r}$ & . & II & . & . & . & . & . & . & . & - \\
\hline Dactylis glomerata & . & . & + & +.2 & . & . & . & . & +.2 & . & . & . & II & . & . & . & . & . & . & . & - \\
\hline Phleum pratense & . & . & + & + & . & . & . & +.2 & . & . & . & . & II & . & . & . & . & . & . & . & - \\
\hline \multicolumn{22}{|l|}{ VII. Ch. Artemisietea vulgaris } \\
\hline Artemisia vulgaris & $+^{\circ}$ & + & 1.1 & 2.1 & 1.1 & +.2 & $+^{\circ}$ & $+^{\circ}$ & 1.1 & . & . & + & $\mathrm{V}$ & + & + & $+^{\circ}$ & + & 1.1 & $+^{\circ}$ & $+^{\circ}$ & V \\
\hline Urtica dioica & $+^{o}$ & $+^{\circ}$ & $+^{\circ}$ & + & . & . & $\mathrm{r}$ & . & . & . & + & + & III & . & . & +.2 & + & + & 1.2 & $+^{\circ}$ & IV \\
\hline Elymus repens & . & + & + & + & + & . & + & + & + & . & . & $\mathrm{r}$ & IV & + & 1.2 & . & . & +.2 & . & . & III \\
\hline Cirsium arvense & $+^{\circ}$ & $+^{\circ}$ & $+^{\circ}$ & . & $\mathrm{r}$ & . & $+^{\circ}$ & . & $\mathrm{r}^{\circ}$ & $\mathrm{r}^{\circ}$ & . & . & III & . & . & . & . & . & $\mathrm{r}^{\circ}$ & . & I \\
\hline Galium aparine & + & $\mathrm{r}$ & + & +.2 & . & + & . & . & . & . & . & +.2 & III & $\mathrm{r}$ & . & . & . & . & . & . & I \\
\hline Melilotus alba & + & 1.1 & 1.1 & + & . & . & . & + & + & . & + & . & III & . & . & . & . & . & . & . & - \\
\hline Tanacetum vulgare & $+^{\circ}$ & $+^{\circ}$ & $+^{\circ}$ & 1.1 & $\mathrm{r}^{\circ}$ & . & . & $+^{\circ}$ & . & . & . & . & III & . & . & . & . & . & . & . & - \\
\hline Cirsium vulgare & $\mathrm{r}^{\circ}$ & $+^{\circ}$ & $+^{\circ}$ & + & . & . & . & $\mathrm{r}^{\circ}$ & . & . & . & . & III & . & . & . & . & $\mathrm{r}^{\circ}$ & . & . & I \\
\hline Medicago lupulina & 2.1 & 1.2 & 1.2 & 2.3 & $\mathrm{r}^{\circ}$ & . & . & . & . & . & + & . & III & . & . & . & . & . & . & . & - \\
\hline Myosoton aquaticum & $+^{\circ}$ & $+^{\circ}$ & $+^{\circ}$ & . & . & . & . & . & . & . & . & . & II & . & . & . & . & . & . & $+^{\circ}$ & I \\
\hline Arctium tomentosum & $+^{\circ}$ & $+^{\circ}$ & $+^{\circ}$ & . & . & . & $+^{\circ}$ & . & . & . & . & . & II & . & . & . & . & . & . & $+^{\circ}$ & I \\
\hline Galeopsis tetrahit & + & $\mathrm{r}$ & + & . & . & . & . & . & $\mathrm{r}$ & . & . & . & II & . & . & . & . & . & . & . & - \\
\hline Lapsana communis & $\mathrm{r}$ & . & . & . & . & . & . & . & . & $\mathrm{r}$ & . & . & $\mathrm{I}$ & $\mathrm{r}$ & . & . & $\mathrm{r}^{\circ}$ & . & . & . & II \\
\hline Rumex obtusifolius & $\mathrm{r}^{\circ}$ & . & . & $\mathrm{r}$ & . & . & $\mathrm{r}^{\circ}$ & . & . & . & . & . & II & . & . & . & $\mathrm{r}$ & . & . & . & $\mathrm{I}$ \\
\hline \multicolumn{22}{|l|}{ VIII. Others } \\
\hline Brassica napus & + & . & . & + & . & $\mathrm{r}^{\circ}$ & $\mathrm{r}$ & . & . & . & . & . & II & + & + & . & . & . & . & . & II \\
\hline Bromus carinatus & + & + & . & $\mathrm{r}$ & . & +.2 & . & . & . & . & . & $\mathrm{r}$ & III & . & . & . & . & . & . & . & - \\
\hline Polygonum hydropiper & . & . & . & . & . & . & . & . & . & . & . & . & - & . & . & . & . & . & 1.2 & 1.2 & II \\
\hline Rorippa palustris & . & . & $\mathrm{r}$ & . & . & . & . & . & . & . & . & . & I & . & . & . & . & . & . & +.2 & $\mathrm{I}$ \\
\hline Polygonum lapathifolium s.s. & . & . & . & . & . & . & . & . & . & . & . & . & - & . & . & . & . & . & . & 2.2 & I \\
\hline Bidens tripartita & . & . & . & . & . & . & . & . & . & . & . & . & - & . & . & . & . & . & . & $\mathrm{r}$ & I \\
\hline Juncus bufonius & + & + & . & +.2 & . & . & . & . & . & . & . & . & II & . & . & . & . & . & $+^{\circ}$ & 1.2 & II \\
\hline Barbarea vulgaris & + & + & 2.1 & + & . & . & . & . & . & . & . & . & II & . & . & . & . & . & . & . & - \\
\hline Chenopodium glaucum & + & . & $\mathrm{r}$ & . & . & . & . & . & . & . & . & . & I & . & . & . & 1.2 & . & . & . & I \\
\hline Epilobium tetragonum & . & $\mathrm{r}$ & + & . & . & . & $\mathrm{r}$ & . & . & . & . & . & II & . & . & . & . & . & . & . & - \\
\hline Stachys sylvatica & + & $\mathrm{r}^{\circ}$ & $\mathrm{r}^{\circ}$ & . & . & . & . & . & . & . & . & . & II & . & . & . & . & . & . & . & - \\
\hline
\end{tabular}

Abbreviations: K - Królewo, Ł - Łącko, Ms - Marszewo, Mw - Marwice, N - Noskowo, Op - Opalewo, Rz - Rzyszczewo, St - Staniewice, $\mathrm{Zg}$ - Zielonagóra

Sporadic taxa: III. Asperugo procumbens 11(+); Bromus sterilis 11(+); Cannabis sativa var. spontanea 11(+); Chenopodium ficifolium 1(+), 3(1.1); Epilobium ciliatum 1(+), 4(r); Sisymbrium loeselii 5(r); IV. Amaranthus retroflexus 12(+); Anagallis arvensis 1(+); Anchusa arvensis $1(+), 2(+), 16(\mathrm{r})$; Anthemis arvensis 2(+), 4(r); Avena fatua $7(+), 13(+)$; Bromus secalinus $8(+)$; Chenopodium hybridum 11(+), 12(+); Euphorbia helioscopia 12(+); Fumaria officinalis 5(+), 10(+); Papaver dubium 4(+), 5(+); P. rhoeas 1(+); Scleranthus annuus 1(r); Sinapis arvensis 1(+), 8(+); VI. Agrostis stolonifera ssp. prorepens 7(+), 9(+), 17(+); A. gigantea 3(+), 9(+); Alopecurus geniculatus 4(+), 5(+); Arrhenatherum elatius 4(+); Festuca pratensis 8(+), 9(+); Geranium molle 3(r); Juncus compressus 15(1.2); J. tenuis 15(+); Leontodon autumnalis 2(+), 19(r); Odontites serotina s.s. 2(+); Poa palustris 3(+); Rumex acetosa 4(r); Stellaria graminea 4(+); Trifolium hybridum s.s. 1(+), 4(+); Veronica serpyllifolia 1(r); Vicia cracca 4(+), 7(+); VII. Anthriscus sylvestris 1(+), 4(r); Arctium lappa 15(+); A. minus 11(+), 15(+); Armoracia rusticana 6(+); Ballota nigra s.s. 11(+); Berteroa incana 3(+); Carduus crispus $9(\mathrm{r})$; Chelidonium majus 3(r); Convolvulus arvensis $12(2.1)$; Dipsacus sylvestris 3(r), 15(r); Epilobium hirsutum 1(+), 3(r); Equisetum arvense 7(r), 9(+); Galeopsis pubescens 10(r); Geum urbanum 2(+), 3(r); Glechoma hederacea 17(+); Heracleum sibiricum 4(r); Melandrium album 4(+); Rubus caesius 7(1.2); Stachys palustris 10(r); Torilis japonica 3(r), 7(r); Tussillago farfara 5(+); VIII. Arenaria serpyllifolia 2(r), 4(+); Barbula convoluta 2(+), 3(+); Brachythecium rutabulum 1(+), 2(+); Bryum sp. 1(1.2), 2(1,2); Ceratodon purpureus 4(2.3), 16(+); Gnaphalium uliginosum 5(r), 7(+); Phleum hubbardii 1(r), 2(+); Triticum vulgare 1(+), 5(+); Agrostis capillaris 4(+); Atriplex prostrata ssp. latifolia 8(r); Avena sativa $7(+)$; Barbarea stricta 4(r); Borago officinalis 1(+); Chenopodium rubrum 3(r); Epilobium parviflorum 16(r); Galeopsis ladanum 1(+); Hordeum vulgare 10(+); Phascum cuspidatum 6(+); Polygonum amphibium fo. terrestre 7(+); Plagiomnium sp. 16(+); Sambucus nigra juv. 2(+); Scrophularia nodosa 3(r), 19(r); Secale cereale 10(+); Solanum tuberosum $10(+)$; Syntrichia ruralis $2(+)$; Trifolium arvense $4(+)$. 


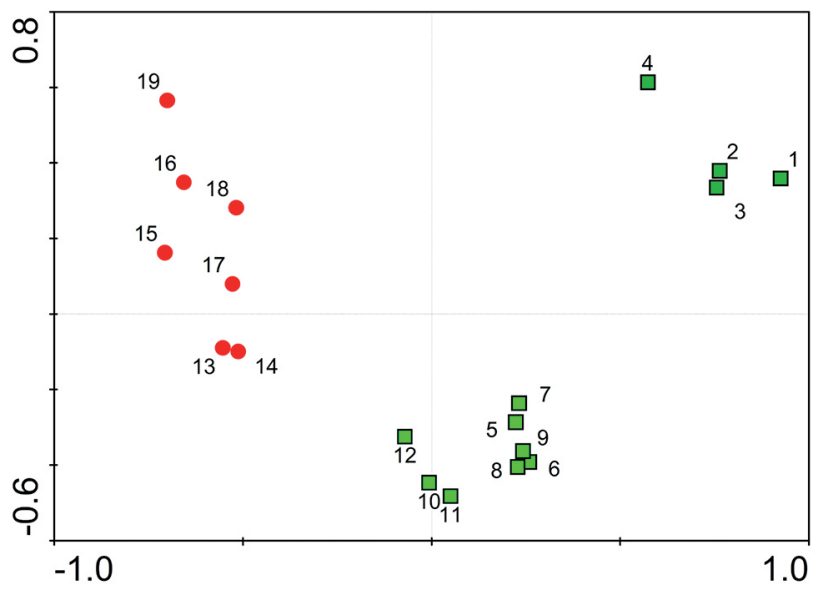

Fig. 6. PCA ordination diagram of relevés of both studied communities

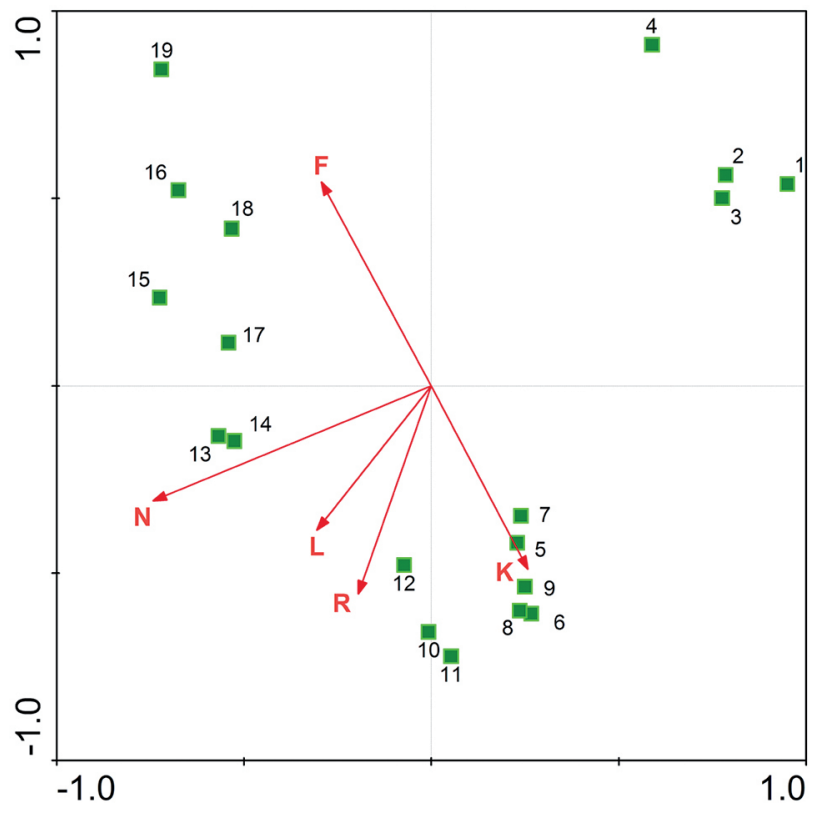

Fig. 7. Passive projection of mean EIV on the PCA ordination diagram of relevés of both studied communities: F - soil moisture, $\mathrm{K}$ - continentality, L - light, $\mathrm{N}$ - soil productivity, $\mathrm{R}$ - soil reaction

The second ordination axis shows differences between Matricario-Anthemidetum cotulae and Matricarietum discoideo-recutitae medicaginetosum in relation to Matricarietum discoideo-recutitae typicum in their plant preferences toward soil humidity and general continentality. Plants constituting Matricario-Anthemidetum cotulae show higher requirements toward humidity, whereas those of Matricarietum discoideo-recutitae typicum higher indices of continentality.

\section{DISCUSSION}

The published materials on Matricarietum discoideo-recutitae since the first description of this unit by JAROLímeK et al. (1997) are still very scarce (total 29 relevés, including 12 published in this paper). The controversial issue is the floristic richness of phyto- coenoses. The mean number of species in one relevé in the original table (JAROLímeK et al. 1997) equals 16 (6-28), in the table of ČARNI (2005), containing only two relevés, it is even lower ( 7 and 10), whereas in our table it reaches 71 in the richer subassociation and 34 in the poorer typical one. In our plots much richer is the representation of characteristic species of Sisymbrietalia and Stellarietea mediae. The typical for this association share of Polygono-Poetea annuae and of Trifolio repentis-Plantaginetalia majoris species is comparable in all materials. Much higher in NW Poland is also share of Artemisietea vulgaris species, they occur however with very low abundances and decreased vitality. This definitely higher floristic richness may result from more diversified spectrum of neighbouring habitats and plant communities, as well as from low intensity of disturbance. In Slovakia all plots were documented from places situated in direct neighbourhood of farm buildings, in the contact with other communities of Malvion neglectae and Matricario-Polygonion arenastri (JAROLímeK et al. 1997). Higher differentiation of habitats occupied by phytocoenoses of Matricarietum discoideo-recutitae in NW Poland results in its differentiation in two subassociations.

The geographic distribution of this community is not fully recognized. It has not been stated so far, for example, from Germany (SCHUbert et al. 2001), Romania (SAnda et al. 2008) and Czech Republic (CHYTRÝ 2009).

Matricario-Anthemidetum cotulae is better documented both in Poland and in other countries of Central Europe, e.g. in Germany (PAssarge 1996, Schubert et al. 2001, Berg et al. 2004), Slovakia (Mucina 1987, JarolímeK et al. 1997) and Czech Republic (CHYTRÝ 2009). Not all authors agree on the syntaxonomy of community with Anthemis cotula. Pender (1990, Table 5, rel. 1-2) includes patches with dominance of Anthemis cotula into Urtico-Malvetum neglectae. In Romania (SANDA et al. 2008) it is currently mentioned as a subassociation Sclerochloo-Polygonetum avicularis (Gams 1927) Soó 1940 anthemidetosum cotulae Vițalariu 1973, within a very broadly approached association placed in the Plantaginetea majoris class.

Both associations are characterized by a high frequency of Polygono-Poetea annuae species in the plots, which is typical trait of the Malvion neglectae communities (Mucina 1987, JarolímeK et al. 1997, Brzeg 2006).

Our data not only confirm the ecological requirements and place of the association Matricarietum discoideo-recutitae in the landscape, published by JARoLímeK et al. (1997) and ČARNi (2005), but broaden the spectrum of its habitats, adding to the courtyards and road margins in the fields, the newly disturbed places, mainly on ruins of destroyed farm buildings. 
All above cited authors (Mucina 1987, Pawlak 1992, Passarge 1996, JarolímeK et al. 1997, Brzeg \& ROSADZIŃSKI 2006, CHYTRÝ 2009) are unanimous in their descriptions of ecological preferences of $M a-$ tricario-Anthemidetum cotulae indicating on villages and on sites moderately disturbed by domestic fowl, enriched in nitrogen of organic origin. Such habitats are situated close to farm buildings, fences and small storage yards and parking places of agricultural machines and vehicles (Fig. 4).

\section{CONCLUSIONS}

The paper gives first documentation of two Malvion neglectae associations from NW Poland. Analogical data from other regions in Poland are scarce, hidden within tables of other communities (Matricario-Anthemidetum cotulae), or not existing in published materials (Matricarietum discoideo-recutitae).

The studies have brought about broadening of knowledge on synecological scale and differentiation of Matricarietum discoideo-recutitae, resulting in description of two new subassociations: $M$. d.-r. medicaginetosum lupulinae and $M$. d.-r. typicum.

Both associations, and especially Matricario-Anthemidetum cotulae, are connected with traditional forms of management in villages and therefore strongly endangered.

\section{REFERENCES}

Berg C., Dengler J., Abdank A., Iserman M., eds (2004): Die Pflanzengesellschaften Mecklenburg-Vorpommerns und ihre Gefährdung - Textband. Weissdorn-Verlag, Jena.

BorHidi A. (2007): Magyarország növénytársulásai. Akadémiai Kiadó, Budapest.

ter BraAk C.J.F., Šmilauer P. (2002): CANOCO ReFERENCE manual and CanoDraw for Windows User's guide: Software for Canonical Community Ordination (version 4.5). Microcomputer Power. Ithaca, New York.

Braun-Blanquet J. (1964): Pflanzensoziologie. Grundzüge der Vegetationskunde. Springer, Wien, New York. http://dx.doi.org/10.1007/9783-7091-8110-2

Brzeg A. (2006): O występowaniu w Wielkopolsce niektórych rzadkich lub mało znanych zbiorowisk synantropijnych. Cz. 1. Badania Fizjograficzne nad Polską Zachodnią B 55: 55-77.

Brzeg A., Rosadziński S. (2006): Zespół rumianu psiego Matricario-Anthemidetum cotulae Dihoru 1995 ex Mucina 1987 w Kotlinie Zasieckiej (Niziny Sasko-Łużyckie). Badania Fizjograficzne nad Polską Zachodnią B 55: 91-98.

Brzeg A., Wojterska M. (2001): Zespoły roślinne Wielkopolski, ich stan poznania i zagrożenie. In: M. Wojterska (ed.). Szata roślinna Wielkopolski i
Pojezierza Południowopomorskiego. Przewodnik sesji terenowych 52. Zjazdu PTB. Poznań, 24-28 września 2001. Bogucki Wyd. Nauk., Poznań: 39-110.

ČARNI A. (2005): Vegetation of trampled habitats in the Prekmurje region (NE Slovenia). Hacquetia 4/2: 151-159.

ChytrÝ M., ed. (2009): Vegetace České republiky. 2. Ruderální, plevelová, skalní a sut'ová vegetace. Vegetation of the Czech Republic. 2. Ruderal, Weed, Rock and Scree Vegetation. Academia, Praha.

Dihoru G. (1975): Invelișul vegetal din Muntele Siriu. Edit. Acad. R.S.R., Bucureşti.

Ellenberg H., Weber H. E., Düll R., Wirth W., WerNer W., PAulissen D. (1992): Zeigerwerte von Pflanzen in Mitteleuropa. 2nd ed. Scripta Geobotanica 18: 1-258.

Jarolímek I., Zaliberová M., Mucina L., Mochnacký S. (1997): Rastlinné spoločenstvá Slovenska. 2. Synantropná vegetácia. Veda, Bratislava.

Mirek Z., PięKoś-Mirkowa H., ZająC A., ZająC M. (2002): Flowering plants and pteridophytes of Poland. A checklist. Vol. 1. Biodiversity of Poland. - Krytyczna lista roślin naczyniowych Polski. T. 1. Różnorodność biologiczna Polski. W. Szafer Institute of Botany, Polish Academy of Sciences, Kraków.

Mucina L. (1987): The ruderal vegetation of the northwestern part of the Podunajská nižina Lowland. 5. Malvion neglectae. Folia Geobotanica et Phytotaxonomica 22(1): 1-24.

Ochyra R., ŻARnowiec J., Bednarek-Ochyra $\mathrm{H}$. (2003): Census Catalogue of Polish Mosses. Vol. 3. Biodiversity of Poland. - Katalog mchów Polski. T. 3. Różnorodność biologiczna Polski. W. Szafer Institute of Botany, Polish Academy of Sciences, Kraków.

PAssarge H. (1996): Pflanzengesellschaften Nordostdeutschlands. I. Hydro- und Therophytosa. J. Cramer, Berlin, Stuttgart.

PAWLAK G. (1992): Roślinność synantropijna Konińskiego Zagłębia Węgla Brunatnego i jego obrzeży. PhD thesis. Zakład Ekologii Roślin i Ochrony Środowiska Uniwersytetu im. A. Mickiewicza, Poznań.

Pender K. (1990): Lasy obszaru Wzgórz Strzelińskich i ich zbiorowiska zastępcze. 2. Zbiorowiska zastępcze. Acta Universitatis Wratislaviensis 1156, Prace Botaniczne 44: 141-255.

RatyńsKa H., Wojterska M., Brzeg A., KoŁacz M. (2010): Multimedialna encyklopedia zbiorowisk roślinnych Polski Ver. 1.1. NFOSiGW, UKW, IETI, Bydgoszcz.

RutKowski L. (2004): Klucz do oznaczania roślin naczyniowych Polski niżowej. Wyd. 2. Wydawnictwo Naukowe PWN, Warszawa. 
Sanda V., Öllerer K., Burescu P. (2008): Fitocenozele din România. Sintaxonomie, structură, dinamică şi evoluție. Ars docendi, Universitatea din Bucureşti.

Schubert R., Herdam H., Weinitschke H., Frank J. (2001): Prodromus der Pflanzengesellschaften Sachsen-Anhalts. Mitteilungen zur floristischen Kartierung Sachsen-Anhalt, Sonderheft 2. Botanisches Verein Sachsen-Anhalt e.V. Halle (Saale).

SowA R. (1971): Flora i roślinne zbiorowiska ruderalne na obszarze województwa łódzkiego ze szczególnym uwzględnieniem miast i miasteczek. Uniwersytet Łódzki, Łódź.
TRuchan M., SobIsz Z. (2007): Vascular flora of a former manor parks in Niebędzino, Pogorzelice and Żelazkowo (the Łeba Valley). Roczniki AR Poznań 86, Botanika-Steciana 11: 65-70.

WojTersKa M., RATYŃSKA H., RĄCZKOWSKi W. (2007): Współczesna struktura krajobrazu roślinnego średniowiecznych założeń osadniczych w zachodniej Polsce. In: W. Dreszer (ed.). Sztuka projektowania krajobrazu. Materiały z międzyuczelnianej konferencji „Architektura krajobrazu kulturowego". ASP, Poznań.

ZająC A. (1978): Założenia metodyczne „Atlasu rozmieszczenia roślin naczyniowych w Polsce”. Wiadomości Botaniczne 22(3): 145-155. 